\title{
Sparse Asymmetric Connectors in Communication Networks ${ }^{\star}$
}

\author{
R. Ahlswede and H. Aydinian
}

\begin{abstract}
An $(n, N, d)$-connector is an acyclic digraph with $n$ inputs and $N$ outputs in which for any injective mapping of input vertices into output vertices there exist $n$ vertex disjoint paths of length $d$ joining each input to its corresponding output. We consider the problem of construction of sparse $(n, N, 2)$-connectors (depth 2 connectors) when $n \ll$ $N$. The probabilistic argument in [1] shows the existence of $(n, N, 2)-$ connectors of size (number of edges) $\mathrm{O}(N)$ if $n \leq N^{1 / 2-\varepsilon}, \varepsilon>0$. However, the known explicit constructions with $n \leq \sqrt{N}$ in [6],[1],[2] are of size $\mathrm{O}(N \sqrt{n})$. Here we present a simple combinatorial construction for $(n, N, 2)$-connectors of size $\mathrm{O}\left(N \log _{2} n\right)$. We also consider depth 2 faulttolerant connectors under arc or node failures.
\end{abstract}

Keywords: connector, rearrangeable network, fault-tolerant connector.

\section{Introduction}

An $(n, N)$-network is a directed acyclic graph with $n$ distinguished vertices called inputs and $N$ other distinguished vertices called outputs. All other vertices are called links. The size of the network is the number of edges, and the depth is the length of the longest path from an input to an output.

An $(n, N, d)$-connector (also called a rearrangeable network) is a network of depth $d(n \leq N)$, such that for every injective mapping of input vertices into output vertices there exist $n$ vertex disjoint paths joining each input to its corresponding output. Connectors are useful architectures for parallel machines. Their study started from pioneering works [13], [14], [8], [3], in connection with practical problems arisen in designing of switching networks for telephone traffic.

Symmetric connectors, i.e. $(n, n, d)$-connectors are well studied. Pippenger and Yao [11] obtained lower and upper bounds for the size of an $(n, n, d)-$ connector: $\Omega\left(n^{1+1 / d}\right)$ and $\mathrm{O}\left(n^{1+1 / d}(\log n)^{1 / d}\right)$, respectively. The best known explicit construction for odd depth $2 i+1$ is $\mathrm{O}\left(n^{1+1 /(i+1)}\right)$, due to Pippenger [12]. Hwang and Richards [7] gave an explicit construction for depth 2 connectors of size $\mathrm{O}\left(n^{5 / 3}\right)$. For asymmetric connectors Oruc [10] gave constructions for depth $\Omega\left(\log _{2} N+\log _{2} n\right)$ of size $\mathrm{O}\left((N+n) \log _{2} n\right)$ and for depth $\Omega\left(\log _{2} N+\log _{2}^{2} n\right)$ of size $\mathrm{O}\left(N+n \log _{2} n\right)$. Hwang and Richards [7] gave an explicit construction for $(n, N, 2)$-connectors of size $(1+o(1)) N \sqrt{n}$ if $n \leq \sqrt{N}$. Baltz, Jäger and Srivastav [1], [2] showed by a probabilistic argument the existence of $(n, N, 2)$-connectors

\footnotetext{
* Supported by DFG-Schwerpunkt Nr.1126 "Algorithmik großer und komplexer Netzwerke".
} 
of size $\mathrm{O}(N)$, if $n \leq N^{1 / 2-\varepsilon}, \varepsilon>0$. They also extended the result of Hwang and Richards and improved it by a constant factor.

We are interested in the case when $n \ll N$. Such connectors with $d=2$ are of particular interest (refering to $[1,2]$ ) in the design of sparse electronic switches. A challenging problem is to construct linear-sized $(n, N, 2)$-connectors. In this paper we show how to construct $(n, N, 2)$-connectors of size $\mathrm{O}\left(N \log _{2} n\right)$.

Let us consider the following standard approach to build connectors of depth 2 .

Suppose the vertex set $V=\mathcal{I} \cup \mathcal{L} \cup \mathcal{O}$ of a graph $G=(V, E)$ is partitioned into input vertices $\mathcal{I}$ with $|\mathcal{I}|=n$, link vertices $\mathcal{L}$ with $|\mathcal{L}|=L$ and output vertices $\mathcal{O}$ with $|\mathcal{O}|=N$. Then one can easily see that $G$ is an $(n, N, 2)$-connector if the following two conditions are satisfied.

$\mathrm{C} 1: \mathcal{I}$ and $\mathcal{L}$ are completely connected, that is inputs and links form a complete bipartite graph.

$\mathrm{C} 2:|\Gamma(S)| \geq|S|$ holds for every $S \subset \mathcal{O}$ with $|S| \leq n$, i.e. the Hall's condition (shortly $H$-condition) is fulfilled for every $n$-set of output vertices $\mathcal{O}$.

In this paper we consider connectors satisfying conditions C1,C2. In Section 3 we give a simple explicit construction for such connectors of size $(1+o(1)) N \log _{2} n$ for all $n<N^{1 / \sqrt{\log _{2} N}}$.

In Section 4 we consider reliable or fault-tolerant connectors. An $(n, N, 2)-$ connector is called $t$-edge fault-tolerant if in spite of any $t$ or less edge failures it still remains an $(n, N, 2)$-connector. Correspondingly, it is called $t$-vertex faulttolerant if $t$ or less vertex failures are admissible in the connector.

We show that our construction can be used to obtain $t$-edge/vertex faulttolerant connectors.

\section{Preliminary}

We need some notation and definitions from extremal set theory. Let $[n]:=$ $\{1, \ldots, n\}$. Given $n, k \in \mathbb{N}$ we denote

$$
2^{[n]}=\{A: A \subset[n]\}, \quad\left(\begin{array}{c}
{[n]} \\
k
\end{array}\right)=\left\{A \in 2^{[n]}:|A|=k\right\} .
$$

For a family $\mathcal{A} \subset\left(\begin{array}{c}{[n]} \\ k\end{array}\right)$ we use the notation $\|\mathcal{A}\|:=\left|\cup_{A \in \mathcal{A}} A\right|$. The shadow of $\mathcal{A} \subset\left(\begin{array}{c}{[n]} \\ k\end{array}\right)$ is defined by

$$
\partial(\mathcal{A})=\left\{F \subset\left(\begin{array}{c}
{[n]} \\
k-1
\end{array}\right): \exists A \in \mathcal{A}: F \subset A\right\} .
$$

Define also the colex order for the $k$-sets of $\mathbb{N}$ denoted by $\left(\begin{array}{c}\mathbb{N} \\ k\end{array}\right)$ : For $A, B \in\left(\begin{array}{l}\mathbb{N} \\ k\end{array}\right)$

$$
A \prec B \quad \Leftrightarrow \quad \max ((A \backslash B) \cup(B \backslash A)) \in B .
$$

Let $\mathcal{C}(k, m)$ denote the first $m$ members of $\left(\begin{array}{l}\mathbb{N} \\ k\end{array}\right)$ in the colex ordering.

The following fact is well known (see [4] or [5]). Every positive integer $m$ can be uniquely represented in the following form called the $k$-cascade representation of $m$ : 


$$
m=\left(\begin{array}{c}
a_{k} \\
k
\end{array}\right)+\left(\begin{array}{c}
a_{k-1} \\
k-1
\end{array}\right)+\cdots+\left(\begin{array}{c}
a_{t} \\
t
\end{array}\right)
$$

with $a_{k}>a_{k-1}>\cdots>a_{t} \geq t \geq 1$.

Recall now the well-known Kruskal-Katona Theorem (see [4],[5]).

Theorem KK. Let $\mathcal{A}$ be a family of $k$-sets with $|\mathcal{A}|=m$ and let $m$ have the cascade representation (2.1).

Then we have

$$
|\partial(\mathcal{A})| \geq|\partial(\mathcal{C}(k, m))|
$$

or equivalently

$$
|\partial(\mathcal{A})| \geq\left(\begin{array}{c}
a_{k} \\
k-1
\end{array}\right)+\cdots+\left(\begin{array}{c}
a_{t} \\
t-1
\end{array}\right) .
$$

For our purposes we need the following

Corollary KK (see [5]). Let $\mathcal{A} \subset\left(\begin{array}{l}\mathbb{N} \\ k\end{array}\right)$ with

$$
|\mathcal{A}| \leq\left(\begin{array}{c}
2 k-1 \\
k
\end{array}\right)+\left(\begin{array}{c}
2 k-3 \\
k-1
\end{array}\right)+\cdots+\left(\begin{array}{l}
3 \\
2
\end{array}\right)+\left(\begin{array}{l}
1 \\
1
\end{array}\right) .
$$

Then $|\partial(\mathcal{A})| \geq|\mathcal{A}|$ and the bound (2.3) is best possible.

\section{The Construction}

Given $n<N$, let $k$ be the smallest integer such that

$$
n \leq\left(\begin{array}{c}
2 k-1 \\
k
\end{array}\right)+\left(\begin{array}{c}
2 k-3 \\
k-1
\end{array}\right)+\cdots+\left(\begin{array}{l}
3 \\
2
\end{array}\right)+\left(\begin{array}{l}
1 \\
1
\end{array}\right)
$$

We take the $k$-cascade representation of

$$
N=\left(\begin{array}{c}
a_{k} \\
k
\end{array}\right)+\left(\begin{array}{c}
a_{k-1} \\
k-1
\end{array}\right)+\cdots+\left(\begin{array}{c}
a_{t} \\
t
\end{array}\right)
$$

and put

$$
L=\left(\begin{array}{c}
a_{k} \\
k-1
\end{array}\right)+\left(\begin{array}{c}
a_{k-1} \\
k-2
\end{array}\right)+\cdots+\left(\begin{array}{c}
a_{t} \\
t-1
\end{array}\right) .
$$

Consider now the set $\mathcal{C}(k, N)$. It is known (see [4],[5]) that

$$
\partial(\mathcal{C}(k, N))=\mathcal{C}(k-1, \mid \partial(\mathcal{C}(k, N) \mid)),
$$

moreover, $|\partial(\mathcal{C}(k, N))|=L$.

Our construction now is straightforward. The vertex set of our graph $G=$ $(V, E)$ is $V=\mathcal{I} \cup \mathcal{L} \cup \mathcal{O}$ with $|\mathcal{I}|=n,|\mathcal{L}|=L,|\mathcal{O}|=N$.

$\mathrm{C} 1$. The inputs $\mathcal{I}$ and links $\mathcal{L}$ form a complete bipartite graph. We identify the outputs with $\mathcal{C}(k, N)$, and the links with $\partial(\mathcal{C}(k, N))$. Thus the vertices of $\mathcal{O}$ and $\mathcal{L}$ correspond respectively to the initial $N k$-sets and $L(k-1)$-sets of $\mathbb{N}$ in the colex ordering. The edges between links and outputs are defined in a natural way, by inclusion. Clearly, each vertex of $\mathcal{O}$ has $k$ neighbors $(k$ subsets from $\left(\begin{array}{c}\mathbb{N} \\ k-1\end{array}\right)$ ). 
C2. Corollary KK implies that $|\Gamma(S)|>|S|$ for every subset $S \subset \mathcal{O}$ with $|S| \leq n$. Thus we have the following

Proposition. The described construction (called $\partial$-construction) gives an $(n, N)$ connector of size

$$
|E|=n L+N k \text {. }
$$

Let us estimate $|E|$ in terms of parameters $n$ and $N$. By (3.1) we have

$$
\Theta\left(\log _{2} n\right)=k \leq \log _{2} n .
$$

By (3.2) and (3.3) we have

$$
N=\Theta\left(\left(\begin{array}{c}
a_{k} \\
k
\end{array}\right)\right), \quad L=\mathrm{O}\left(\frac{k}{a_{k}} N\right),
$$

where $a_{k} \geq \frac{k}{e}\left(\begin{array}{c}a_{k} \\ k\end{array}\right)^{1 / k}$ and hence

$$
a_{k}=\Omega\left(\log _{2} n N^{1 / \log _{2} n}\right) .
$$

Finally, (3.4),(3.5),(3.6) and (3.7) give

$$
|E|=n L+N k=N \log _{2} n\left(\mathrm{O}\left(\frac{n}{a_{k}}\right)+1\right) .
$$

If now $n=\mathrm{O}\left(\log _{2} n N^{1 / \log _{2} n}\right)$, then

$$
|E|=\mathrm{O}\left(N \log _{2} n\right) .
$$

In particular, we have the following

Theorem 1. For all $N$ and $n=O\left(N^{1 / \sqrt{\log _{2} N}}\right)$ there are explicitly constructible $(n, N, 2)$-connectors of size $(1+o(1)) N \log _{2} n$.

\section{Fault-Tolerant Connectors}

In this section we show that by $\partial$-construction we can also obtain $t$-faulttolerant connectors.

Theorem 2. Let $G=(V, E)$ be an $(n, N)$-connector given by $\partial$-construction, and let $k$ be the degree of output vertices defined from $\left(\begin{array}{c}2 k-3 \\ k\end{array}\right)<n \leq\left(\begin{array}{c}2 k-1 \\ k\end{array}\right)$. Then $G$ is a $(k-1)$-edge fault-tolerant connector.

To prove the theorem we need the following

Lemma 1. Let $\mathcal{A} \subset\left(\begin{array}{l}\mathbb{N} \\ k\end{array}\right)$. Then

$$
|\partial \mathcal{A}|-|\mathcal{A}| \geq k-1
$$

if $(a)|\mathcal{A}| \leq\left(\begin{array}{c}2 k-1 \\ k\end{array}\right)-k+1$, or $(b)\left(\begin{array}{c}2 k-1 \\ k\end{array}\right)-k+1<|\mathcal{A}| \leq\left(\begin{array}{c}2 k-1 \\ k\end{array}\right)$ and $\|\mathcal{A}\| \neq 2 k-1$. 
Proof. Case (a) can be easily derived from Theorem KK by simple calculations. Case (b): The cascade representation of $|\mathcal{A}|$ for this case is

$$
|\mathcal{A}|=\left(\begin{array}{c}
2 k-2 \\
k
\end{array}\right)+\left(\begin{array}{c}
2 k-3 \\
k-1
\end{array}\right)+\cdots+\left(\begin{array}{c}
a_{1} \\
1
\end{array}\right)
$$

where $1 \leq a_{1} \leq k-1$, or

$$
|\mathcal{A}|=\left(\begin{array}{c}
2 k-1 \\
k
\end{array}\right)
$$

Hence by Theorem KK

$$
|\partial(\mathcal{A})| \geq\left(\begin{array}{c}
2 k-2 \\
k-1
\end{array}\right)+\left(\begin{array}{c}
2 k-3 \\
k-2
\end{array}\right)+\cdots+\left(\begin{array}{c}
k \\
1
\end{array}\right)+\left(\begin{array}{c}
a_{1} \\
0
\end{array}\right)=\left(\begin{array}{c}
2 k-1 \\
k
\end{array}\right) .
$$

Note that $\|\mathcal{A}\|>2 k-1$ (since $\|\mathcal{A}\| \neq 2 k-1)$. We use now the result in Moers [9] which generalizes the Kruskal-Katona Theorem. In our special case, $\|\mathcal{A}\|>2 k-1$, it says that the minimum of $|\partial \mathcal{A}|$ is attained for $\|\mathcal{A}\|=2 k$. Moreover, an optimal family achieving the minimum is

$$
\mathcal{A}^{*}=\mathcal{C}(k,|\mathcal{A}|-1) \cup(\{1, \ldots, k-1,2 k\}) .
$$

Observe now that

$$
\left|\partial\left(\mathcal{A}^{*}\right)\right|=\left(\begin{array}{c}
2 k-1 \\
k
\end{array}\right)+k-1
$$

\section{Proof of Theorem 2}

Let $G=(V, E)$ be an $(n, N, 2)$-connector given by $\partial$-construction. Given an injective mapping $\phi: \mathcal{I} \rightarrow \mathcal{O}$, let $\mathcal{M}=\phi(\mathcal{I}) \subset \mathcal{O}$. Define also $\mathcal{L}^{\prime}=\Gamma(\mathcal{M})$. Let now $G^{\prime}$ be the subgraph of $G$ induced by the vertices $\mathcal{I} \cup \mathcal{L}^{\prime} \cup \mathcal{M}$. Let also $E_{1}$ and $E_{2}$ be the edge sets of $G^{\prime}$ induced by $\mathcal{I} \cup \mathcal{L}^{\prime}$ and $\mathcal{L}^{\prime} \cup \mathcal{M}$, respectively. Suppose now $t(t \leq k-1)$ edge-failures have occurred in $G^{\prime}$. Since $\mathcal{I} \cup \mathcal{L}^{\prime}$ is a complete bipartite graph, we note that the "worst" case is that all faults are from $E_{2}$. Thus it is sufficient to show that after deletion of $t$ edges in $E_{2}$ the resulting graph satisfies the $H$-condition.

Suppose now $S \subseteq \mathcal{M}$. Remind that $n=|\mathcal{I}|=|\mathcal{M}| \leq\left(\begin{array}{c}2 k-1 \\ k\end{array}\right)$. Let $\mathcal{A} \subset\left(\begin{array}{l}\mathbb{N} \\ k\end{array}\right)$ be the family identified with $S$. If $\mathcal{A}$ satisfies one of the conditions of Lemma 1 , then (4.1) means that $|\Gamma(S)|-|S| \geq k-1$. This clearly implies that the removal of any $k-1$ link-vertices (and hence any $k-1$ edges) from $G^{\prime}$ results in a graph, where the output vertices satisfy the $H$-condition. Thus it remains to consider the case $|\mathcal{A}|>\left(\begin{array}{c}2 k-1 \\ k\end{array}\right)-k+1$ with $\|\mathcal{A}\|=2 k-1$.

This case is easier to examine in terms of the incidence matrix $H$ for $\mathcal{A}$ and $\partial(\mathcal{A})$ (equivalently, the adjacency matrix of the corresponding bipartite graph induced by $\Gamma(S) \cup S)$. Let the columns of $H$ be labeled by elements of $\mathcal{A}$ and the rows by elements of $\partial(\mathcal{A})$.

Without loss of generality we may assume that $\mathcal{A} \subset\left(\begin{array}{c}{[2 k-1]} \\ k-1\end{array}\right)$. Suppose first that $\mathcal{A}=\left(\begin{array}{c}{[2 k-1]} \\ k\end{array}\right)$ and consider the corresponding incidence matrix $H$ of size $\left(\begin{array}{c}2 k-1 \\ k\end{array}\right) \times\left(\begin{array}{c}2 k-1 \\ k\end{array}\right)$. 
Each column and each row of $H$ contains exactly $k$ ones. Delete now any $k-i-1(1 \leq i \leq k-1)$ columns in $H$.

\section{Lemma 2.}

(i) Every row in the resulting matrix $H^{\prime}$ contains at least $i+1$ ones.

(ii) Every two rows of $H^{\prime}$ contain together at least $k+i$ ones.

Proof. (i) is obvious. (ii) follows from the fact that any two rows have at most one 1 in common and hence two rows in $H^{\prime}$ have at least $2 k-(k-i)=k+i$ ones. Thus the incidence matrix $H^{\prime}$ of every family $\mathcal{A}$ with $|\mathcal{A}|=\left(\begin{array}{c}2 k-1 \\ k\end{array}\right)-k+1+i$ has the properties (i) and (ii).

Exchange now any $t \leq k-1$ ones in $H^{\prime}$ into zeros (which corresponds to deletion of $t$ edges in $E_{2}$ ), obtaining a new matrix $H^{*}$. In view of (i) and (ii) $H^{*}$ contains at most one all-zero row. Thus the sum of all columns of $H^{*}$ contains at most one zero coordinate if $i<k-1$ and no zero coordinate if $i=k-1$. This implies that for the corresponding bipartite graph $G^{*}$ (with $t$ deleted edges) $|\Gamma(S)| \geq|S|$ holds for every set of output vertices $S$ with $\left(\begin{array}{c}2 k-1 \\ k\end{array}\right)-k+1<|S| \leq\left(\begin{array}{c}2 k-1 \\ k\end{array}\right)$.

This completes the proof of Theorem 2 .

Clearly, if $G$ is a $t$-vertex fault-tolerant $(n, N)$-connector, then it is also a $t$-edge fault-tolerant $(n, N)$-connector. Thus in general we can speak about $t-$ fault-tolerant connectors under edge-vertex failures.

The following statement directly follows from the proof of Theorem 2 .

Corollary 1. The $\partial$-construction gives a $(k-1)$-fault-tolerant $(n, N)$-connector if

$$
\left(\begin{array}{c}
2 k-3 \\
k-1
\end{array}\right)<n \leq\left(\begin{array}{c}
2 k-1 \\
k
\end{array}\right)-k+1
$$

\section{A Direction of Further Research}

Our main tool are results on shadows of subfamilies of the family of subsets of a finite set, which is a poset by the inclusion relation. It is conceivable that shadow properties of other posets give better results.

\section{References}

1. A. Baltz, G. Jäger, and A. Srivastav, Elementary constructions of sparse asymmetric connectors, Proceedings of the 23rd Conference on Foundations of Software Technology and Theoretical Computer Science (FSTTCS), Mumbai, India, LNCS 2914, 13-22, 2003.

2. A. Baltz, G. Jäger, and A. Srivastav, Constructions of sparse asymmetric connectors with number theoretic methods, Networks, Vol. 45, No. 3, 119-124, 2005.

3. V.E. Beneś, Optimal rearrangeable multistage connecting networks, Bell System Tech. J. 43, 1641-1656, 1964.

4. B. Bollobas, Combinatorics, Cambridge University Press, 1986. 
5. K. Engel, Sperner Theory, Cambridge University Press, 1997.

6. C. Clos, A study of non-blocking switching networks, Bell System Tech. J. 32, 406-424, 1953.

7. P. Feldman, J. Friedman, and N. Pippenger, Wide-sense nonblocking networks, SIAM J. Discr. Math. 1, $158-173,1988$.

8. F.K. Hwang and G.W. Richards, A two-stage rearrangeable broadcast switching network, IEEE Trans. on Communications 33, 1025-1035, 1985.

9. M. Moers, A generalization of a theorem of Kruskal, Graphs and Combinatorics 1, 167-183, 1985.

10. A.Y. Oruc, A study of permutation networks: some generalizations and tradeoffs, J. of Parall. and Distr. Comput., 359-366, 1994.

11. N. Pippenger and A.C. Yao, On rearangeable networks with limited depth, SIAM J. Algebraic Discrete Methods 3, 411-417, 1982.

12. N. Pippenger, On rearangeable and nonblocking switching networks, J. Comput. System Sci. 17, 145-162, 1987.

13. C.E. Shannon, Memory requirements in a telephone exchange, Bell System Tech. J. 29, 343-349, 1950.

14. D. Slepian, Two theorems on a particular crossbar switching network, unpublished manuscript, 1952. 